\title{
Static black holes in equilibrium with matter: nonlinear equation of state
}

\author{
Oleg B. Zaslavskii \\ Astronomical Institute of Kharkov V.N. Karazin National University, \\ 35 Sumskaya Street., Kharkov, 61022, Ukrain€*
}

\begin{abstract}
We consider a spherically symmetric black hole in equilibrium with surrounding classical matter that is characterized by a nonlinear dependence of the radial pressure $p_{r}$ on the density $\rho$. We examine under which requirements such an equilibrium is possible. It is shown that if the radial and transverse pressures are equal (Pascal perfect fluid), equation of state should be approximately linear near the horizon. The corresponding restriction on $\frac{d p_{r}}{d \rho}$ is a direct generalization of the result, previously found for an exactly linear equation of state. In the anisotropic case there is no restriction on equation of state but the horizon should be simple (nondegenerate).

PACS numbers: 04.70.Dy, 04.70.Bw, 04.40.Nr
\end{abstract}

\section{INTRODUCTION}

In real physical circumstances, any black hole is surrounded by matter. If some special conditions imposed on the equation of state are fulfilled, an equilibrium between a black hole and matter can occur, so that there is no collapse, the metric being static or stationary. As a result, some sort of hair appears around a black hole. If matter is described by the linear equation of state, only quite definite discrete set of such a hair enumerated by two integers is possible [1]. Meanwhile, in general nonlinear equations of state look more realistic. Therefore, of interest is the question: which types of matter are compatible with the static black hole horizon in the nonlinear case? Below, we suggest an answer for the simplest but

*Electronic address: ozaslav@kharkov.ua 
physically relevant case of spherically symmetric configurations, thus extending the results of [1].

Consider a spherically symmetric metric which can be written in the form

$$
d s^{2}=-A d t^{2}+\frac{d u^{2}}{A}+r^{2}\left(d \theta^{2}+\sin \theta^{2} d \phi^{2}\right) .
$$

Here $u$ is called a quasiglobal coordinate, and the location of the event horizon corresponds to the outmost zero of $A$. It is convenient to use the combination of 00 and 11 Einstein equations, the conservation law and 11 equation. They read, respectively,

$$
\begin{gathered}
2 A \frac{r^{\prime \prime}}{r}=-8 \pi\left(p_{r}+\rho\right), \\
p_{r}^{\prime}+\frac{2 r^{\prime}}{r}\left(p_{r}-p_{\perp}\right)+\frac{A^{\prime}}{2 A}\left(p_{r}+\rho\right)=0 \\
\frac{1}{r^{2}}\left(-1+A^{\prime} r r^{\prime}+A r^{\prime 2}\right)=8 \pi p_{r}
\end{gathered}
$$

where the prime denotes derivation with respect to $u$. Without the loss of generality, we can assume that on the horizon $u=0$. We are interested in the conditions (if any) of which equation of state should obey for the configuration to be regular in the vicinity of the horizon. The latter means, by definition, that the metric coefficients and the energy density should be analytical functions of $u$ in the vicinity of the horizon. In particular, near the horizon of the $\mathrm{n}$-th order $A \sim u^{n}$ where $n>0$ is an integer.

\section{EQUATION OF STATE: GENERAL ASYMPTOTIC FORM}

We assume the general form of the relationship between the density and radial pressure

$$
p_{r}=f(\rho)
$$

First of all, we are going to elucidate which general asymptotics of $f(\rho)$ is admissible near the horizon. On the horizon itself we must have [2] (p. 207), [3]

$$
\rho_{h}+p_{h r}=0
$$


where the subscript " $h$ " means that the corresponding quantity is calculated on the horizon. Let now for $\rho \rightarrow \rho_{h}$

$$
f(\rho) \approx-\rho_{h}+w_{h}\left(\rho-\rho_{h}\right)^{\alpha}
$$

where $w_{h}$ and $\alpha>0$ are constants.

In what follows, the properties of the system depend crucially on whether or not $p_{r}-p_{\perp}=$ 0 . In the linear case $f=w \rho$ considered in [1] it was unimportant. Indeed, because of the assumption $\left|p_{\perp}\right| / \rho<\infty$ [1] (which is physically reasonable and is more weak than the energy dominant condition) the simple estimate shows that the second term in (3) has the same order as $\rho$ and can be neglected near the horizon where $\rho \rightarrow 0$ (see [1] for details). Meanwhile, now $\rho_{h} \neq 0$ in general, so one should be careful. Therefore, we consider separately the isotropic case

$$
p_{r}=p
$$

(which corresponds to the perfect fluid) and the anisotropic one $p_{r} \neq p$.

\section{ISOTROPIC CASE}

Now, the second term in (3) cancels. The equation can be integrated to give

$$
A=A_{0} \exp (-2 z), z=\int \frac{d \rho}{f+\rho} \frac{d f}{d \rho} .
$$

If $\alpha>1$, one can obtain from (9) or (3) and (7) that near the horizon $z \sim\left(\rho-\rho_{h}\right)^{\alpha-1}+$ const, $A \approx$ const $\neq 0$ contrary to the fact that on the horizon we must have $A\left(\rho_{h}\right)=0$. Thus, matter with the equation of state under discussion having $\alpha>1$ cannot support a horizon.

If $\alpha<1$, we obtain

$$
A \sim\left(\rho-\rho_{h}\right)^{-2 \alpha}
$$

Instead of the horizon, we have a singularity, so this choice of $\alpha$ also should be rejected.

Thus, only the case $\alpha=1$ is admissible: near the horizon any equation of state should behave as a linear one.

Let now $\alpha=1$. Then, we assume also that near the horizon

$$
\rho-\rho_{h}=B_{k} u^{k}+o\left(u^{k}\right)
$$


where $k>0$ is an integer, and $B_{k}$ is a constant. Then, collecting in (3) the main contributions of each term which have the order $k-1$ and equating the corresponding coefficient to zero, we obtain that

$$
w_{h}=\left(\frac{d f}{d \rho}\right)_{\rho=\rho_{h}}=-\frac{n}{n+2 k}<0,\left|w_{h}\right|<1
$$

that generalizes the previous observation made in [1] for the particular case $f=w \rho$ with a constant $w$.

The same holds true if, instead of the exact equality (8), we have near the horizon

$$
\left|p_{r}-p_{\perp}\right| \leq C\left|\rho-\rho_{h}\right|
$$

where $C$ is some constant.

\section{ANISOTROPIC CASE}

Now we assume that the conditions (8), (13) are violated.

As we want to derive some general model-independent conclusions, we suppose that, generically, $r_{h}^{\prime} \neq 0$. Then, in eq. (3) the term of the zero order appears due to the difference $p_{r}-p_{\perp}$. To satisfy eq. (3) and compensate this term, we must have $k=1$, with

$$
\left.\frac{B_{1}}{2}\left[w_{h}(n+2)+n\right)\right]+\frac{2 r_{h}^{\prime}}{r_{h}}\left(p_{r}-p_{\perp}\right)_{\mid u=u_{h}}=0
$$

One can determine the coefficient $B_{1}$ from this equation, so there is no restriction on the form of equation of state. Moreover, the quantity $w_{h}$ may have any sign, so this case includes

matter with $\frac{\partial p_{r}}{\partial \rho}>0$ that can be of interest bearing in mind the stability issue. Further, as $k=1$ and it follows from (2) that $k \geq n$ (assuming also that $r_{h}^{\prime \prime} \neq 0$ ), we have also $n=1$ (a simple horizon).

\section{VACUUM FLUID AND NEAR-HORIZON EXPANSION: COMPARISON}

Near the horizon, taking the expansion $\rho=\rho_{h}+\tilde{\rho}$ and $p_{r}=-\rho_{h}+\tilde{p}_{r}$ with small $\tilde{\rho}$ and $\tilde{p}_{r}$, one can represent approximately the total stress-energy tensor as

$$
T_{\mu}^{\nu}=\tilde{T}_{\mu}^{\nu}+T_{(v a c) \mu}^{\nu}
$$


where $\tilde{T}_{\mu}^{\nu}$ is linear with respect to $\tilde{\rho}$ and $p_{(v a c)} r=-\rho_{v a c} \equiv-\rho_{h}$. Thus, by definition, $T_{(v a c) \mu}^{\nu}$ has the form of so-called dark (vacuum) fluid. Therefore, it is instructive to compare the results for 1) a generic nonlinear equation of state and case 2) when from the very beginning there is a mixture of two noninteracting terms - the matter with the linear equation of state and the vacuum fluid [1] In the isotropic case $p_{r}=p_{\perp}$ (perfect fluid) the results are the same, with (12) slightly generalizing the corresponding relation for the linear equation of state [1]. However, if $p_{r} \neq p_{\perp}$ they are qualitatively different. Namely, in case 2 eq. (12) still holds whereas in a case 1 there is no restriction on the equation of state at all. Instead, there is a restriction on the type of the horizon (only $n=1$ is admissible) in case 1 whereas there is no such a restriction in case 2 [1]. This difference between the two cases can be attributed to the fact that in case 1 one cannot split the total stress-energy tensor to two non-interacting parts, so that the zero-order term contribution the second term in (15) enters the equation for $\tilde{p}_{r}$ and $\tilde{\rho}$ in $(\underline{3})$.

It is also worth noting that in [1] (where $\rho_{h}=p_{h r}=0$ ) eq. (44) was used to distinct the possible types of the horizon when the vacuum field is present or absent. Now, it is irrelevant since, in general, $p_{h r} \neq 0$ without any additional vacuum fluid and any type of the horizon - simple $(n=1)$ or degenerate $(n \geq 2)$ is possible. For the case $n \geq 2$, only the first term in the left hand side of (41) survives, so $p_{h r}=-\frac{1}{8 \pi r_{h}^{2}}$.

\section{CONCLUSION}

Thus, the results of [1] are extended to the arbitrary nonlinear equations of state. It turned out that the properties of equation of state necessary for the possibility of matter to be in equilibrium with the horizon of a static spherically symmetric black hole depend crucially on whether or not the matter represents the perfect fluid. In the first case, any admissible equation of state should behave as effectively linear near the horizon, higher or fractional powers in the expansion like (7) are excluded. Further, there is a restriction on the first derivative $\frac{d p_{r}}{d \rho}$ on the horizon that generalizes the corresponding result for the linear case 1] directly. However, if the radial and transversal pressures do not coincide, the existence of the horizon does not impose any restrictions on the form of equation of state. Instead, it 
restricts strongly the type of the horizon which is shown to be simple only. Although any stress-energy tensor near the horizon is approximately equal to the vacuum contribution

plus small corrections, the results are qualitatively different from [1] due to the effective interaction between both components.

One can rephrase these results by saying that the Pascal perfect fluid admits only discrete set of hair but does not restrict the order of the horizon, whereas generic anisotropic matter allows any hair but only simple horizons.

All restrictions discussed in the present work are model ndependent. In addition, any concrete model can, obviously, add some special restrictions.

\section{ACKNOWLEDGEMENT}

I thank Roman Konoplya for a stimulating question on the seminar that entailed writing this work. I am grateful to the Institute for Theoretical Astrophysics of Tübingen University where this work started, for hospitality and warm atmosphere.

I also thank Kirill Bronnikov for reading the manuscript and useful comments.

[1] K A Bronnikov and O B Zaslavskii. Phys. Rev. D 78, 021501(R) (2008).

[2] V. P. Frolov and I. D. Novikov, Black Hole Physics: Basic Concepts and New Developments, (Kluwer Academic, Amsterdam, 1998).

[3] A. J. M. Medved, D. Martin, and M. Visser, Class. Quant. Grav. 21, 3111 (2004). 